\title{
Insegurança alimentar e nutricional e fatores associados em famílias do Núcleo Rural Agrícola Lamarão, no Distrito Federal
}

\author{
Food and nutritional insecurity and associated factors \\ among families in the Rural Settlement of Lamarão, \\ Distrito Federal
}

\section{Inseguridad alimentaria y nutrición y factores asociados en familias del centro Rural Agrícola Lamarão, en el Distrito Federal}

Carla Madeira Marquito BASTOS ${ }^{1}$

Anelise Rizzolo de Oliveira PINHEIRO²

Muriel Bauermann GUBERT ${ }^{3}$

\section{RESUMO}

Segurança alimentar e nutricional (SAN) é o direito ao acesso regular e permanente a alimentos de qualidade e em quantidade suficiente, não comprometendo outras necessidades essenciais. Este estudo analisou a prevalência de insegurança alimentar e nutricional (ISAN) e seus fatores associados em famílias residentes em um núcleo rural agrícola, no Distrito Federal. Trata-se de estudo transversal com amostra sistemática, com intervalo zero, sendo composta por 75 domicílios. A prevalência de insegurança alimentar e nutricional nas famílias foi estimada por meio da Escala Brasileira de Insegurança Alimentar (EBIA). Variáveis socioeconômicas e demográficas também foram avaliadas. O estudo encontrou $50,6 \%$ de ISAN, sendo $17,3 \%$ moderada e grave. Todas as casas são de alvenaria, com água encanada, energia elétrica e coleta de lixo. Dos domicílios com esgotamento sanitário, $12 \%$ eram contemplados com a rede de saneamento básico. A maior parte dos domicílios era própria (82,7\%). Havia 29,3\% das famílias beneficiárias do Programa Bolsa Família. A classificação econômica familiar predominante esteve nas classes C/D/E (87,3\%). Houve associação significativa entre os níveis de SAN e tipo de domicílio, chefe do domicílio e

1 Nutricionista, graduada pela Universidade de Brasília. Mestranda em Nutrição Social, do Programa de Pós Graduação em Nutrição Humana da Universidade de Brasília. E-mail: carlabastos207@hotmail.com

2 Possui graduação em Nutrição pela Universidade Federal de Pelotas, especialização em saúde pública, Mestrado em Saúde Pública pela Universidade Federal de Santa Catarina e Doutorado em Política Social pela Universidade de Brasília. É professora adjunta do Departamento de Nutrição da Faculdade de Saúde da Universidade de Brasília.

3 Nutricionista, formada pela Universidade de Brasília, Mestre em Ciências da Saúde e Doutora em Ciências da Saúde. Especialista em Atenção Primária à Saúde e em Gestão de Pólíticas de Alimentação e Nutrição pela FIOCRUZ. É professora do Curso de Nutrição da Universidade de Brasília (UnB) 
consumo de hortaliças, frutas, leite e cereais. A prevalência de ISAN entre as famílias do Lamarão demonstra uma possível limitação dessas famílias em relação ao acesso físico e financeiro e à disponibilidade de alimentos com qualidade nutritiva. É importante um olhar aprofundado sobre a garantia da SAN em comunidades rurais e necessário se considerar as associações entre os fatores socioeconômicos, demográficos e de consumo alimentar, que determinam a ocorrência dos diagnósticos de SAN na população.

Palavras chave: insegurança alimentar e nutricional; população rural; consumo alimentar.

\section{ABSTRACT}

Food and Nutritional Security is the right to regular and permanent access to enough and good quality food, without compromising other essential needs. This study examined the prevalence of alimentary and nutritional insecurity and associated factors among families in a rural settlement in Distrito Federal. This is a cross-sectional study, involving a systematic sample, with a time interval equals to zero, composed by 75 households. The prevalence of unsafe nutrition among those families was established by the "Escala Brasileira de Insegurança Alimentar". Socioeconomic and demographic variables were also considered. The study found $50,6 \%$ of alimentary and nutritional insecurity, with $17,3 \%$ classified as moderate and severe. All houses are made of bricks and equipped with piped water, electricity and waste collection; only $12 \%$ of homes have access to sewage disposal. Most of it is composed by residence-owned houses $(82,7 \%)$. Around $29 \%$ of the families were in receipt of "Bolsa Família". The prevailing economic classification involved families from classes $\mathrm{C} / \mathrm{D} / \mathrm{E}(87,3 \%)$. There was a significant association between levels of food security and type of home, family leader and the consumption of green leafy vegetables, fruits, milk and cereals. The prevalence of food insecurity among families from Lamarão demonstrates a limitation related to physical and financial access, as well as availability of nutritional food. It's important to examine thoroughly food security among rural population and it is necessary to consider the associations among socioeconomic, demographic and food consumption factors that determine the occurrence of the diagnosis of food security in the population.

Keywords: food insecurity; rural population; food consumption.

\section{RESUMEN}

Seguridad alimentaria y nutricional es el derecho de acceso regular y permanente a la comida de calidad y en cantidad suficiente, sin comprometer otras necesidades fundamentales. Este estudio examinó la prevalencia de la inseguridad alimentaria y nutricional y sus factores asociados en las familias que viven en núcleo rural agrícola, en el Distrito Federal. Es un estudio de corte transversal con muestreo sistemático, con rango cero, se compone de 75 hogares. La prevalencia de la inseguridad alimentaria y nutricional de las familias se calcula mediante la "Escala Brasileira de Insegurança Alimentar”. Variables socioeconómicas y demográficas también fueron evaluados. 
El estudio encontró 50,6\% de inseguridad alimentaria, siendo 17,3\% moderada/severa. Todas las viviendas son de mampostería, con agua corriente, electricidad y la recolección de basura. De todas las casas, el 12\% tienen saneamiento. La mayoría de los hogares eran propios (82,7\%). El 29,3\% de las familias reciben el "Programa Bolsa Família". La clasificación económica predominante es clases $\mathrm{C} / \mathrm{D} / \mathrm{E}(87,3 \%)$. Se observó una asociación significativa entre niveles de seguridad alimentaria y tipo de hogar, jefe del hogar y consumo de verduras, frutas, leche y cereales. Prevalencia de inseguridad alimentaria entre las familias de Lamarão demuestra una posible limitación en relación con el acceso físico y financiero y la disponibilidad de alimentos de calidad nutricional. Es importante considerar la garantía de seguridad alimentaria en las poblaciones rurales y es necesario considerar las asociaciones entre factores socioeconómicos, demográficos y de consumo alimentaria, que determinan la aparición de los diagnósticos de seguridad alimentaria en la población.

Palabras clave: inseguridad alimentaria; población rural; consumo de alimentos.

\section{INTRODUÇÃO}

Segurança alimentar e nutricional (SAN) se dá quando é realizado o direito ao acesso regular e permanente a alimentos de qualidade, em quantidade suficiente, sem comprometer o alcance de outras necessidades essenciais, tendo como base práticas alimentares promotoras da saúde que respeitem a diversidade cultural e que sejam ambiental, cultural, econômica e socialmente sustentáveis ${ }^{1}$.

Já a insegurança alimentar e nutricional (ISAN) acontece quando o alimento não está disponível, ou quando a capacidade de acessar esses alimentos é incerta ou limitada, tanto em termos qualitativos quanto em termos quantitativos; esse reflexo pode variar do medo de a família não conseguir o alimento até a fome $e^{2,3}$. A insegurança alimentar relaciona-se, normalmente, com a vulnerabilidade social, sendo resultado de uma combinação de situações causadoras de degradação na qualidade de vida como desemprego e renda familiar insuficiente, produção e distribuição dos alimentos de forma irregular e o afronte aos direitos sociais que devem ser assegurados às famílias ${ }^{2,4,5}$.

Na conjunção brasileira atual, a insegurança alimentar tem se manifestado como resultado de consequências de uma alimentação inadequada e não saudável, em função da falta de acesso físico e/ou financeiro aos alimentos, ou mesmo da falta de informações sobre o que é uma alimentação adequada e saudável ${ }^{5,6}$. Esse é o perfil alimentar e nutricional paradoxal e incoerente que afeta a população brasileira, na qual se encontra a obesidade (grande problema de saúde pública), ao lado da desnutrição e das deficiências de micronutrientes, como formas mais graves de manifestação da insegurança alimentar $6,7,8$.

A prevalência de insegurança alimentar e nutricional nas zonas rurais, verificada pela Pesquisa Nacional por Amostra de Domicílios (PNAD), tanto em 2004 quanto em 2009, sinaliza diferenças espaciais no Brasil. As comparações entre os domicílios particulares mostram sempre níveis de 
insegurança alimentar e nutricional nos domicílios rurais superiores aos domicílios urbanos. Em 2004, a insegurança das áreas rurais brasileiras era de 43,6\%, contra 33,3\% das áreas urbanas. Já em 2009 houve diminuição dessa insegurança nas áreas rurais (35,1\%). Ainda assim, os dados apontam prevalências de ISAN moderada e grave maior nas áreas rurais quando comparadas às áreas urbanas brasileiras ${ }^{2}$.

Estudos nas zonas rurais brasileiras demonstram que fatores socioeconômicos e demográficos, como o total de moradores no domicílio, número de menores de 18 anos, escolaridade e o sexo do chefe do domicílio e a baixa renda familiar mensal são associados à insegurança alimentar e nutricional nesse contexto $2,10,11$.

A respeito do consumo alimentar, a última Pesquisa de Orçamentos Familiares (POF), de 2008/2009, constatou o consumo excessivo de gorduras saturadas, açúcar, sal, e a carência de fibras na alimentação brasileira em geral, além de baixo consumo de frutas e hortaliças ${ }^{12}$. Nas áreas rurais, esse mesmo comportamento alimentar já é descrito em famílias diagnosticadas com insegurança alimentar e nutricional ${ }^{11,13}$.

Especificamente nos domicílios rurais brasileiros, a insegurança alimentar e nutricional, aliada ao baixo consumo de frutas e hortaliças, tem sido associada principalmente às condições socioeconômicas escassas das famílias para a compra de alimentos adequados e saudáveis e ao acesso insuficiente a terra, à renda, a equipamentos e meios para uma produção agrícola adequada e permanente $\mathrm{e}^{7,11,14}$.

Nas zonas rurais e agrícolas, fatores sociais, econômicos e de acesso a bens e serviços podem auxiliar planejamentos e criações de políticas de garantia da segurança alimentar e nutricional, possibilitando um desenvolvimento rural mais adequado ${ }^{15,16}$. No debate sobre a garantia da SAN, faz-se necessária a inclusão de ações relativas às populações rurais, visto que a situação histórica e social brasileira tradicionalmente as negligencia ${ }^{7,16,17}$.

No Distrito Federal, o contexto rural é diferenciado do Brasil como um todo, caracterizado por forte presença de atributos urbanos, influenciando as situações cotidianas ${ }^{18,19}$.

A organização territorial do início da construção do Distrito Federal e de Brasília tinha por objetivo a designação específica de uma área rural estruturada, com a capacidade de produção de alimentos, a qual daria surgimento aos então chamados “núcleos rurais de Brasília”. O projeto inicial buscava transformar o espaço urbano com atividades urbanas, e não agrícolas, em um espaço rural com atividades agrícolas. Entretanto, o processo de construção e de ocupação desse território rural não ocorreu como o esperado e o programado. O espaço "planejado" para ser o meio rural no DF acabou com características locais únicas e singulares, pelas influências dos impactos urbanos ${ }^{18,19,20}$.

Existem estudos que avaliam as condições de SAN em comunidades rurais e agrícolas 
brasileiras $^{2,7,11,16,17}$, no entanto, são escassas as informações sobre essas realidades no DF, pois a maior parte dos estudos é voltada para os diagnósticos urbanos ${ }^{20}$.

Os dados mais recentes sobre a situação de segurança alimentar e nutricional dos domicílios urbanos do Distrito Federal afirmam que a população local tem adquirido mais alimentos inadequados e não saudáveis, influenciando os casos de excesso de peso. Observa-se ainda a presença de ISAN nas famílias, confirmando que esse diagnóstico está associado aos fatores socioeconômicos, como a renda domiciliar e a composição familiar ${ }^{2,20}$.

Sendo assim, o presente estudo tem o propósito de analisar a prevalência de insegurança alimentar e nutricional e seus fatores associados em famílias residentes no Núcleo Rural Agrícola Lamarão, localizado em uma zona rural e agrícola do Paranoá - Distrito Federal.

\section{METODOLOGIA}

Trata-se de um estudo transversal, com amostra sistematizada, realizado com as famílias residentes no Núcleo Rural Agrícola Lamarão, situado na zona rural e agrícola da Região Administrativa do Paranoá, no Distrito Federal.

O Núcleo Rural Agrícola Lamarão está localizado na área denominada Programa de Assentamento Dirigido do Distrito Federal (PAD-DF), dentro do Paranoá. O PAD-DF foi um programa governamental da década de 70, que procurava ocupar o cerrado com a agricultura do agronegócio. Ao todo, o PAD-DF é formado por seis comunidades agrícolas e rurais, que produzem soja, milho, feijão, hortaliças, frutas, trigo ${ }^{21,22}$.

A comunidade rural do Lamarão é composta por uma população de, aproximadamente, 330 habitantes, no arranjo de 127 famílias, distribuídas em uma agrovila e em chácaras. São habitantes vindos, principalmente, do Nordeste brasileiro. $\mathrm{Na}$ agrovila residem moradores atraídos por possibilidades de empregos na região do PAD-DF e na Fazenda Malunga ${ }^{\circledR}$ (localizada dentro do território do Lamarão), uma fazenda que comercializa produtos orgânicos. Nas chácaras localizam-se algumas famílias de agricultores rurais ${ }^{21,22}$. O Lamarão participou de um programa de desenvolvimento tecnológico destinado à agricultura e hoje possui luz elétrica e asfalto nas ruas principais. No local existe um centro comunitário, uma pequena igreja, um campo gramado de futebol e um comércio simples. A comunidade rural conta com o apoio e atendimento da equipe da Estratégia de Saúde da Família, do Centro de Saúde número 8, da Região Administrativa do Paranoá 22 .

A amostra inicial foi calculada em 56 domicílios, considerando o $n$ populacional de 127 domicílios, e a prevalência do evento insegurança alimentar grave em 7\%, com erro máximo de estimativa de 5\%. A sistematização seguiu a ordem de disposição geográfica dos domicílios, com intervalo de zero, para evitar perdas significativas. Ao final, a amostra foi composta por 75 
domicílios.

Como critérios de inclusão do estudo, o entrevistado deveria residir no domicílio selecionado, ter, no mínimo, 18 anos de idade, ser reconhecedor da dinâmica familiar e estar disposto a participar da pesquisa.

A coleta de dados da pesquisa ocorreu de janeiro a março de 2013, aos finais de semana. A equipe de trabalho era formada por alunos e professores da Faculdade de Ciências da Saúde (FS), da Universidade de Brasília (UnB), integrantes do Projeto de Extensão do Pró-Saúde (Ministério da Saúde) chamado "Construindo Ambientes Saudáveis - Análise dos seus Reflexos na Saúde, Nutrição e Ambiente das Famílias Acompanhadas pela Estratégia Saúde da Família, Comunidade Lamarão - DF", do qual esse estudo teve origem. Além disso, houve apoio e auxílio da Equipe de Saúde da Família (ESF), composta por uma médica, uma enfermeira e um agente comunitário de saúde.

Um questionário foi utilizado, contendo questões com variáveis socioeconômicas e demográficas, níveis de insegurança alimentar e nutricional, estado nutricional de indivíduos adultos e consumo alimentar semanal das famílias.

As variáveis socioeconômicas e demográficas avaliadas eram referentes a: características do entrevistado, características dos domicílios, composição familiar e características de seus chefes, tempo de residência da família na comunidade, recebimento de benefício social do Programa Bolsa Família (PBF) e classificação econômica familiar.

$\mathrm{Na}$ caracterização do entrevistado, foram perguntados idade, sexo, estado civil (casado ou em união estável; solteiro/divorciado/viúvo) e escolaridade (analfabeto/ensino fundamental completo; ensino médio completo/ensino superior completo).

$\mathrm{Na}$ caracterização dos domicílios, foram avaliados o tipo de domicílio (próprio ou cedido; alugado), o material utilizado para a construção do domicílio (alvenaria) e a presença de água encanada, energia elétrica, coleta de lixo e saneamento básico.

Na composição familiar foram investigados o número de moradores no domicílio (famílias com até três pessoas; famílias com quatro ou mais pessoas) e famílias com menores de 18 anos no domicílio (famílias sem menores de 18 anos; famílias com até três menores de 18 anos).

A classificação econômica familiar seguiu o Critério de Classificação Econômica Brasil ${ }^{23}$, o qual utiliza questões que envolvem o poder de compra das famílias, pontuando e classificando-as em classes econômicas, de acordo com a posse de bens duráveis e o grau de instrução do chefe da família. Com a pontuação obtida, as famílias podem ser classificadas em A1, A2, B1, B2, C1, C2, D e E. Para melhor representação e classificação econômica, neste estudo as famílias foram 
agrupadas nas categorias A e B (A/B) e C, D e E (C/D/E).

Além disso, foram conseguidas informações também sobre o sexo do chefe de família (homem ou mulher) e a sua escolaridade (analfabeto/ensino fundamental incompleto; até a quarta série do ensino fundamental; ensino fundamental completo; ensino médio completo/ensino superior completo) segundo a categorização do próprio Critério de Classificação Econômica Brasil. Também para melhor representação e realização do teste estatístico de base binária, a classificação da escolaridade do chefe de família foi agrupada nas categorias analfabeto/ensino fundamental incompleto, até a quarta série do ensino fundamental e ensino fundamental completo (Analfabeto/ Ensino Fundamental) e ensino médio e ensino superior (Ensino Médio/Ensino Superior).

Para caracterização dos níveis de insegurança alimentar e nutricional foi aplicada a Escala Brasileira de Insegurança Alimentar - EBIA, sendo os domicílios considerados seguros ou não de acordo com escore de respostas positivas ${ }^{2,24}$. Os domicílios foram classificados, conforme pontuação obtida, em segurança alimentar (ausência de insegurança) ou em insegurança alimentar. No caso de insegurança alimentar, os domicílios foram classificados segundo os seus três níveis: insegurança leve, moderada e grave ${ }^{2}$. Foram agrupadas as categorias de insegurança alimentar moderada e grave, uma vez que nessas duas classificações os moradores do domicílio (incluindo as crianças) convivem ou já conviveram com alguma restrição quantitativa de alimentos, passando por privação alimentar, no período referido pela escala, de três meses.

Para a realização do teste estatístico de base binária, foram agrupadas as categorias segurança alimentar e insegurança alimentar leve (SAN/ISAN Leve) e insegurança alimentar moderada e insegurança alimentar grave (ISAN Moderada/Grave). Sabe-se que, ao ser feita essa priorização, limita-se o estudo em relação à restrição qualitativa da dieta. Porém, por outro lado, a abordagem da qualidade da alimentação é parcialmente explicada quando se é avaliado o consumo alimentar semanal familiar (qualidade da alimentação das famílias) mediante o uso de um Questionário de Frequência Alimentar (QFA).

Outros estudos brasileiros já trabalharam com esse agrupamento segundo uma analogia entre segurança alimentar, na qual não há qualquer restrição alimentar, e a insegurança alimentar leve, na qual pode ocorrer comprometimento qualitativo da alimentação e preocupação com o acesso aos alimentos. Já nos casos das categorias de insegurança alimentar moderada e insegurança alimentar grave a analogia que ocorre é a do comprometimento da quantidade de alimentos, chegando a casos de fome entre os membros familiares ${ }^{25,26,27}$. Dessa forma, foi escolhida essa junção para se verificar as associações mais quantitativas e serem discutidos os resultados mais significativos.

Optou-se, também, pela realização da avaliação do estado nutricional dos indivíduos adultos, porque a literatura brasileira tem mostrado a relação entre insegurança alimentar e nutricional e o excesso de peso entre adultos $3,5,8,20,28,29$. 
Foi realizada a antropometria apenas do adulto informante, em cada família entrevistada. A aferição do peso corporal utilizou balança portátil com capacidade de até $150 \mathrm{~kg}$ e precisão de 100 gramas. No momento da aferição, os indivíduos estavam descalços, com roupas bem leves e sem qualquer objeto pesado nos bolsos. Para a aferição da altura utilizou-se estadiômetro portátil. Os indivíduos ficavam descalços e encostados à parede, com postura ereta. Todas as aferições antropométricas foram realizadas duas vezes para cada indivíduo, sendo assim minimizados possíveis erros de mensuração, empregando-se a média dos valores em caso de diferenças.

Para a avaliação antropométrica foi utilizado o Índice de Massa Corporal (IMC), com a então classificação do estado nutricional dos indivíduos em baixo peso, eutrófico, sobrepeso e obesidade ${ }^{30}$. Nesse caso, as variáveis sobrepeso e excesso de peso, no momento das associações estatísticas, foram agrupadas na classificação denominada "excesso de peso".

Para avaliação do consumo alimentar, aplicou-se um questionário adaptado, baseado no Questionário de Frequência Alimentar (QFA), do Sistema de Vigilância Alimentar e Nutricional $(\mathrm{SISVAN})^{30}$, para uma estimativa da qualidade da alimentação dessas famílias. Era perguntado ao entrevistado, em relação à respectiva família, a frequência de consumo de certos grupos alimentares como legumes e verduras cozidas e cruas; frutas; feijão e/ou leguminosas; leite e/ou derivados; cereais e pães; tubérculos; carnes; embutidos; biscoitos/bolachas doces e/ou doces; biscoitos/ bolachas salgadas, salgadinhos de pacote; refrigerantes e sucos industrializados.

O QFA é um instrumento de avaliação prático, rápido e de baixo custo, o qual afere o consumo alimentar habitual de populações. Sua estrutura registra a frequência do consumo de certos tipos de alimentos segundo um período de tempo ${ }^{31}$.

A avaliação do consumo foi feita mediante as frequências declaradas para cada grupo alimentar. Utilizaram-se as categorias "não consome" (denominada baixo consumo); consumo entre "uma a quatro vezes na semana" (médio consumo); consumo entre "cinco e sete vezes na semana" (alto consumo). Posteriormente, o consumo alimentar foi classificado em "consumo adequado" (entre uma a sete vezes na semana) e "consumo inadequado" (não consome) para os alimentos considerados saudáveis; e "consumo adequado" (não consome) e "consumo inadequado" (entre uma a sete vezes na semana) para os alimentos considerados não saudáveis. Em relação aos grupos alimentares saudáveis legumes/verduras e frutas, foi considerado o consumo adequado aquele igual ou superior a cinco dias na semana, segundo o recomendado pela literatura brasileira ${ }^{32}$.

Todos os dados foram analisados no Programa SPSS Statistics ${ }^{\circledR}$ (Statistical Package for the Social Sciences) versão 20.0. Foi utilizado o teste de Qui-Quadrado para verificar as possíveis associações entre a situação de segurança alimentar e nutricional e variáveis socioeconômicas e demográficas, estado nutricional e consumo alimentar semanal, sendo considerados significativos os valores de $\mathrm{p} \leq 0,005$. 
A presente pesquisa teve aprovação no Comitê de Ética em Pesquisa, da FS/UnB, com o registro de número 159/11. As famílias participantes assinaram o Termo de Consentimento Livre e Esclarecido.

\section{RESULTADOS E DISCUSSÕES}

Foram entrevistadas 75 famílias (59\% da totalidade dos domicílios) do Núcleo Rural Agrícola Lamarão, compreendendo 283 habitantes.

A Tabela 1 apresenta os dados sobre as características socioeconômicas e demográficas, os níveis de insegurança alimentar e nutricional das famílias e o estado nutricional dos adultos.

A maioria dos entrevistados eram mulheres (72\%), casadas ou em união estável (77,3\%), com o ensino fundamental completo $(58,7 \%)$ e com média de idade de 51 anos.

Foi verificada a existência de insegurança alimentar e nutricional em 50,6\% das famílias, sendo a insegurança leve a forma mais prevalente $(33,3 \%)$. A prevalência de insegurança alimentar

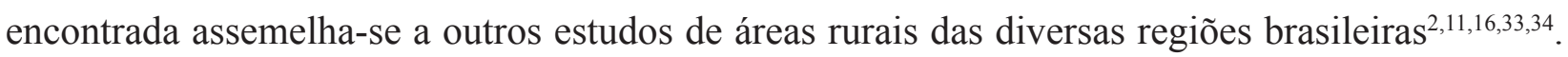
No Distrito Federal, a prevalência de insegurança alimentar em áreas urbanas é de $21,2 \%$, sendo $15,1 \%$ de insegurança leve ${ }^{2}$. A PNAD em 2009 já apontava os contrastes entre os ambientes urbanos e rurais, havendo prevalência de insegurança alimentar maior nas áreas rurais ${ }^{2,34}$.

A garantia da segurança alimentar e nutricional de famílias de áreas rurais brasileiras torna-se cada vez mais importante e necessária, visto que a população rural ainda é negligenciada e sofre as consequências de um inadequado desenvolvimento rural (em muitos casos inexistente) ${ }^{16,17}$. A segurança alimentar e nutricional é um direito destes indivíduos também, estando fundamentada em políticas públicas ${ }^{1}$.

As características socioeconômicas e demográficas indicam as condições de acesso a bens e a serviços básicos de uma residência e sua relação com a saúde da famíliaa, 2, 15,25,35. A insegurança alimentar e nutricional das famílias brasileiras tem sido descrita como resultado de questões socioeconômicas e ambientais inadequadas da realidade populacional, impactando de forma negativa na qualidade de vida e no bem estar dos indivíduos ${ }^{25,34}$. 
$142 / /$

Tabela 1. Caracterização socioeconômica, demográfica e de insegurança alimentar e nutricional das famílias. Núcleo Rural Agrícola Lamarão, Distrito Federal, 2013.

\begin{tabular}{lcc}
\hline & Variáveis & $\mathbf{n}$ \\
\hline Sexo do Entrevistado & & 72 \\
Feminino & 54 & 28 \\
Masculino & 21 & 77,3 \\
Estado Civil do Entrevistado & 58 & 22,7 \\
Casado ou União Estável & 17 & 8 \\
$\quad$ Solteiro/Divorciado/Viúvo & & 58,7 \\
Escolaridade do Entrevistado & 6 & 33,3 \\
$\quad$ Analfabeto & 44 & \\
Ensino Fundamental Completo & 25 & \\
Ensino Médio Completo/Ensino Superior Completo & 51,1 anos \\
Idade do Entrevistado (média) & $(35,8-80,8)$
\end{tabular}

Tipo de Domicílio

\begin{tabular}{lc} 
Próprio ou Cedido & 62 \\
\hline
\end{tabular}

Alugado

Domicílios de Alvenaria com Água Encanada, Energia Elétrica e Coleta de Lixo

Domicílios com Saneamento Básico

Não

Sim

Tempo de Residência da Família na Comunidade

De 1 a 10 anos

Mais de 10 anos

Composição Familiar - $\mathbf{N}^{0}$ de moradores no domicílio

Famílias com até três pessoas

Famílias com quatro pessoas ou mais

Famílias com menores de 18 anos no Domicílio

Famílias sem menores 18 anos

Famílias com até três menores

Recebimento de Benefício Social (Bolsa Família)

$$
\text { Sim }
$$

Não

Chefe de Família

Homem

\section{Escolaridade do Chefe de Família}

Analfabeto/Ensino Fundamental Incompleto

Até Quarta Série do Ensino Fundamental

Ensino Fundamental Completo

Ensino Médio Completo/Ensino Superior Completo

\section{Classificação Econômica Familiar}

$\mathrm{A} / \mathrm{B}$

Níveis de Insegurança Alimentar e Nutricional 


\begin{tabular}{|c|c|c|}
\hline Segurança Alimentar e Nutricional (Ausente) & 37 & 49,4 \\
\hline Insegurança Alimentar e Nutricional Leve & 25 & 33,3 \\
\hline Insegurança Alimentar e Nutricional Moderada e Grave & 13 & 17,3 \\
\hline \multicolumn{3}{|l|}{ Estado Nutricional dos Adultos $\left(n=66^{*}\right)$} \\
\hline Eutrófico & 29 & 43,9 \\
\hline Sobrepeso & 23 & 34,9 \\
\hline Obesidade & 14 & 21,2 \\
\hline Total & 75 & 100 \\
\hline
\end{tabular}

Analisando-se os fatores associados à presença de insegurança alimentar nas famílias da comunidade rural Lamarão, os níveis de segurança alimentar e nutricional e as variáveis "tipo de domicílio" e "chefe do domicílio" foram associados estatisticamente $(\mathrm{p} \leq 0,005)$. Não houve associação estatística entre SAN e as demais variáveis socioeconômicas e demográficas (Tabela 2).

Este estudo apontou que, apesar de estar em uma zona rural, a situação do Lamarão é bem diferenciada das demais realidades no Brasil ${ }^{2,11,16}$ : todas as casas são de alvenaria, com água encanada, energia elétrica e coleta de lixo (Tabela 1). Porém, semelhante aos demais domicílios rurais brasileiros ${ }^{2}$, apenas nove domicílios (12\%) são contemplados com a rede de saneamento básico. Esses dados já haviam sido apresentados anteriormente no trabalho de Carneiro et al. ${ }^{22}$, sendo confirmados com o estudo atual. A maior parte dos domićlios é próprio/cedido $(82,7 \%)$ e $61,3 \%$ das famílias residem há mais de 10 anos na comunidade.

$\mathrm{Na}$ avaliação de segurança alimentar e nutricional, a discussão sobre a presença de saneamento básico nos domicílios é fundamental ${ }^{2,5,8,35}$. Domicílios brasileiros com ISAN são aqueles proporcionalmente menos atendidos pela rede de saneamento básico ${ }^{2}$. No Lamarão, dos 13 domicílios com insegurança moderada/grave, 76,9\% não eram contemplados com o saneamento; porém essa associação não foi significativa (Tabela 2). No estudo de Nascimento et al. ${ }^{16}$, a insegurança alimentar apresentava-se maior quanto pior eram as condições de saneamento.

A maior parte dos domicílios investigados é próprio ou cedido $(82,7 \%)$, havendo associação estatisticamente significativa $(\mathrm{p}=0,005)$ com a situação de SAN da família (Tabela 2). No caso de segurança alimentar/insegurança leve, $87,1 \%$ das famílias residem em domicílios próprios ou cedidos; nos níveis de insegurança moderada/grave, grande parte $(46,2 \%)$ das famílias reside em domicílios alugados. Ou seja, no Lamarão a condição de moradia é um indicador da avaliação da segurança alimentar e nutricional. Quando o domicílio é próprio, gastos como aluguel ou outros tipos de pagamentos similares são reduzidos, podendo ser a renda destinada a outras necessidades básicas, como a despesa com uma alimentação mais adequada e saudável para a família ${ }^{2,36}$.

Das 75 famílias estudadas, 43 (57,3\%) são compostas por quatro ou mais pessoas (Tabela 1), sendo as famílias com insegurança moderada/grave as mais populosas (Tabela 2). Isso mostra uma 
característica das famílias do Lamarão possuírem arranjos familiares com a presença de mais de uma pessoa na residência, seguindo uma tendência das tradicionais famílias brasileiras ${ }^{37}$. Aires et $a l .{ }^{33}$ e Mondini et al. ${ }^{34}$ verificaram associação entre o grau de insegurança alimentar das famílias rurais e o aumento do número de moradores nos domicílios. Na PNAD, os domicílios particulares em insegurança alimentar moderada e grave eram aqueles em que havia concentração maior de residentes ${ }^{2}$.

Com relação à presença ou não de pessoas com menos de 18 anos de idade nos domicílios, 60\% das famílias entrevistadas possuem até três deles em sua composição. No entanto, a presença ou não dos menores de 18 anos não esteve associada à segurança ou insegurança alimentar e nutricional, diferente do observado em trabalhos anteriores com famílias de zonas rurais ${ }^{12,16}$ (Tabela 2).

Segundo Hoffmann ${ }^{38}$, a presença de menores de 18 anos de idade no domicílio influencia e está associada à insegurança alimentar e nutricional. Essa influência, porém, existe apenas quando não há um controle do efeito de outras variáveis envolvidas na determinação da ISAN. Nesses casos, a presença dos menores de 18 anos no domicílio colabora para a diminuição da possibilidade de insegurança alimentar no ambiente, mostrando que essa relação está associada ao número de pessoas na família e não à presença de crianças. No estudo do Lamarão, contudo, essa associação não pôde ser constatada.

Foi verificado também que a maioria das famílias (70,7\%) não é participante do Programa Bolsa Família - PBF (Tabela 1). Das famílias consideradas inseguras moderada/grave, 69,2\% não recebiam o benefício social (Tabela 2). É importante destacar que a transferência condicionada de renda do PBF (mais as ações de saúde) procura reduzir a pobreza, as desigualdades sociais e combater a fome vivenciada pelos membros familiares, portanto, impactando na garantia da segurança alimentar e nutricional ${ }^{26,39}$.

Nesse sentido, é essencial a inclusão das famílias com insegurança alimentar nos programas de transferência de renda. A equipe do Programa Saúde da Família tem papel central na busca e inserção dos beneficiários de programas sociais, em articulação e diálogo com a agenda da assistência social, sendo estratégico o preenchimento do Cadastro Único para Programas Sociais (CadÚnico), uma vez que esse cadastro contém informações e características sobre as famílias de baixa renda no Brasil, conhecendo a realidade socioeconômica dessas famílias ${ }^{40}$.

Os estudos brasileiros têm mostrado associação entre a elevação do grau de insegurança alimentar e famílias participantes de programas sociais, porém essa relação de vício de interpretação pode mostrar uma focalização, porque são exatamente essas famílias as que devem participar do Programa. Vianna \& Segall-Corrêa ${ }^{15}$ constataram, entre as famílias rurais e urbanas de municípios do interior da Paraíba, que, conforme aumentava-se os níveis de insegurança alimentar e nutricional, também aumentava-se a quantidade de famílias em ISAN que eram participantes de programas sociais. Souza et al. ${ }^{9}$, estudando apenas famílias beneficiárias do Programa Bolsa Família, encontrou mais // Tempus, actas de saúde colet, Brasília, 8(2), 133-156, jun, 2014.

ISSN 1982-8829 
de $72 \%$ de insegurança alimentar nas famílias, sendo a metade dessas com insegurança leve. Essa participação de famílias com ISAN em programas sociais é desejada, pois o beneficio social é destinado às famílias em insegurança alimentar e nutricional, já que essas ainda se encontram em condição extrema de vulnerabilidade social, por motivos como: baixa renda familiar, desemprego ou emprego informal do chefe do domicílio, baixa escolaridade do chefe, entre outros ${ }^{26,34,39}$.

O recebimento de benefício social, dessa forma, pode ser considerado um indicador de proteção social. No Lamarão, das 62 famílias consideradas em SAN/ISAN Leve, 29\% eram beneficiárias do Bolsa Família (Tabela 2). Assim, não se pode ignorar a importância que os programas de transferência de renda podem ter para esses indivíduos, auxiliando no desenvolvimento das fontes de renda familiar e na qualidade de vida dos beneficiários ${ }^{38,39}$.

Em relação aos chefes de família, a maior parte dos entrevistados do estudo do Lamarão respondeu serem estes homens $(86,7 \%)$ com o ensino fundamental completo $(41,4 \%)$ (Tabela 1$)$. Nos domicílios em SAN/ISAN Leve, 98,4\% dos chefes de família são homens e 1,6\% são mulheres (Tabela 2). No Lamarão, essa associação entre segurança e insegurança alimentar e gênero do chefe de família foi estatisticamente significativa $(\mathrm{p}=0,000)$.

A literatura brasileira já evidenciou anteriormente essa relação, referindo que o sexo do chefe de família influencia o grau de segurança alimentar e nutricional $2,5,16,27,34,38,41$. Segundo dados da PNAD, em domicílios onde as mulheres eram os chefes das famílias, a situação de ISAN foi mais prevalente ${ }^{2,38}$, e mais elevada nas famílias rurais ${ }^{(34)}$. No próprio Distrito Federal, essa relação também foi encontrada. Ali, domicílios com a responsável mulher apresentavam-se com mais insegurança alimentar ${ }^{(41)}$.

Nos arranjos familiares brasileiros, a maior parte das famílias admite como pessoa de referência o indivíduo do sexo masculino, sendo esse o responsável pelos rendimentos da família e considerado o chefe ${ }^{2,36}$. Quando a mulher é considerada a responsável pelo domicílio, pode ser sugestivo de um domicílio uniparental, sem a presença da figura masculina no domicílio, indicando uma renda familiar ainda menor ${ }^{2,38,41}$.

No Núcleo Rural Lamarão, a classificação socioeconômica familiar mais prevalente foi das classes C/D/E (82,7\%) (Tabela 1), sendo 92,3\% destas famílias com insegurança moderada/grave (Tabela 2). É conhecida essa relação entre os níveis socioeconômicos e a ISAN $5,9,16,27,37$. Nascimento et al. ${ }^{(16)}$ verificou que a insegurança alimentar era mais prevalente nas famílias rurais de classes $\mathrm{C}$, $\mathrm{D}$ e E, em comparação às das áreas urbanas. 
Tabela 2. Situação de segurança alimentar e nutricional segundo as características socioeconômicas e demográficas das famílias, e do estado nutricional dos adultos das famílias. Núcleo Rural Agrícola Lamarão, Distrito Federal, 2013.

\begin{tabular}{|c|c|c|c|c|c|}
\hline \multirow[t]{2}{*}{ Variáveis } & \multicolumn{2}{|c|}{ SAN/ISAN Leve } & \multicolumn{2}{|c|}{ ISAN Moderada/Grave } & \multirow[t]{2}{*}{ p-valor** } \\
\hline & $\mathbf{n}$ & $\%$ & $\mathbf{n}$ & $\%$ & \\
\hline \multicolumn{6}{|l|}{ Tipo de Domicílio } \\
\hline Próprio ou Cedido & 55 & 88,7 & 7 & 53,8 & 0,005 \\
\hline Alugado & 7 & 11,3 & 6 & 46,2 & \\
\hline \multicolumn{6}{|l|}{ Domicílios com Saneamento Básico } \\
\hline Não & 56 & 90,3 & 10 & 76,9 & 0,176 \\
\hline Sim & 6 & 9,7 & 3 & 23,1 & \\
\hline \multicolumn{6}{|l|}{ Tempo de Moradia na Comunidade } \\
\hline De 1 a 10 anos & 21 & 33,9 & 7 & 53,8 & 0,176 \\
\hline Mais de 10 anos & 41 & 66,1 & 6 & 46,2 & \\
\hline \multicolumn{6}{|l|}{ Composição Familiar } \\
\hline Famílias com até três pessoas & 29 & 46,8 & 3 & 23,1 & 0,116 \\
\hline Famílias com quatro pessoas ou mais & 33 & 53,2 & 10 & 76,9 & \\
\hline \multicolumn{6}{|l|}{ Famílias com menores de 18 anos no } \\
\hline $\begin{array}{l}\text { Domicílio } \\
\text { Famílias sem menores } 18 \text { anos }\end{array}$ & 26 & 41,9 & 4 & 30,8 & 0,455 \\
\hline Famílias com até três menores & 36 & 58,1 & 9 & 69,2 & \\
\hline \multicolumn{6}{|l|}{ Recebimento de Benefício Social } \\
\hline $\begin{array}{l}\text { (Bolsa Família) } \\
\text { Sim }\end{array}$ & 18 & 29,0 & 4 & 30,8 & 0,900 \\
\hline Não & 44 & 71,0 & 9 & 69,2 & \\
\hline \multicolumn{6}{|l|}{ Chefe de Família } \\
\hline Homem & 61 & 98,4 & 4 & 30,8 & 0,000 \\
\hline Mulher & 1 & 1,6 & 9 & 69,2 & \\
\hline \multicolumn{6}{|l|}{ Escolaridade do Chefe de Família } \\
\hline Analfabeto/Ensino Fundamental & 53 & 85,5 & 12 & 92,3 & 0,510 \\
\hline Ensino Médio/Ensino Superior & 9 & 14,5 & 1 & 7,7 & \\
\hline \multicolumn{6}{|l|}{ Classificação Econômica Familiar } \\
\hline $\mathrm{A} / \mathrm{B}$ & 12 & 19,4 & 1 & 7,7 & 0,312 \\
\hline $\mathrm{C} / \mathrm{D} / \mathrm{E}$ & 50 & 80,6 & 12 & 92,3 & \\
\hline \multicolumn{6}{|l|}{ Estado Nutricional $(n=66)$} \\
\hline Eutrófico & 22 & 41,5 & 7 & 53,9 & \\
\hline Excesso de Peso & 31 & 58,5 & 6 & 46,1 & 0,464 \\
\hline Total & 62 & 100 & 13 & 100 & \\
\hline
\end{tabular}

**p-valor: $\leq 0,005$

Em relação à avaliação da qualidade da alimentação das famílias, aferida mediante o Questionário de Frequência Alimentar (Tabela 3), constatou-se consumo frequente de feijão e/ou leguminosas e de cereais, além de um elevado consumo (entre uma e sete vezes na semana) dos alimentos considerados não saudáveis: embutidos (65,3\%); biscoitos doces e/ou doces (84\%); biscoitos salgados, salgadinhos de pacote $(85,3 \%)$; refrigerantes $(93,4 \%)$ e suco industrializado $(66,7 \%)$. Foi constatado também o consumo recomendado, segundo a literatura brasileira ${ }^{31}$, entre cinco e sete vezes na semana, de legumes e verduras cruas $(33,4 \%)$, legumes e verduras cozidas $(34,7 \%)$, frutas (44\%) (Tabela 3).

O consumo de carnes pelas famílias estudadas evidencia que, mesmo esse sendo um alimento de elevado custo financeiro para os brasileiros ${ }^{2}$, ele é ainda priorizado, independente da condição de 
SAN das famílias entrevistadas. Esse dado pode estar refletindo um hábito alimentar característico da cultura nordestina presente na região do Lamarão, conforme refere o estudo de Carneiro et al. ${ }^{22}$.

Tabela 3. Caracterização do consumo alimentar semanal das famílias. Núcleo Rural Agrícola Lamarão, Distrito Federal, 2013.

\begin{tabular}{lcc}
\hline \multicolumn{1}{c}{ Variável } & n & \% \\
\hline Legumes e verduras cruas & 10 & 13,3 \\
Não consome & 40 & 53,3 \\
1 a 4 vezes & 25 & 33,4 \\
5 a 7 vezes & & \\
Legumes e verduras cozidas & 7 & 9,3 \\
Não consome & 42 & 56,0 \\
1 a 4 vezes & 26 & 34,7 \\
5 a 7 vezes &
\end{tabular}

\section{Frutas}

Não consome

1 a 4 vezes

5 a 7 vezes

\section{Feijão e/ou Leguminosas}

Não consome

1 a 4 vezes

5 a 7 vezes

\section{Leite e Derivados}

Não consome

1 a 4 vezes

5 a 7 vezes

\section{Cereais e Pães}

Não consome

1 a 4 vezes

5 a 7 vezes

\section{Tubérculos}

Não consome
1 a 4 vezes
5 a 7 vezes

\section{Carnes}

$$
\begin{aligned}
& \text { Não consome } \\
& 1 \text { a } 4 \text { vezes } \\
& 5 \text { a } 7 \text { vezes }
\end{aligned}
$$

62

\section{Embutidos}


Não consome

1 a 4 vezes

5 a 7 vezes
Biscoitos/bolachas doces e/ou doces

Não consome

1 a 4 vezes

5 a 7 vezes

Biscoitos/bolachas salgados, salgadinhos de pacote

Não consome

1 a 4 vezes

5 a 7 vezes

\section{Refrigerantes}

Não consome

1 a 4 vezes

5 a 7 vezes

Suco industrializado

Não consome

1 a 4 vezes

5 a 7 vezes

Total
23,0

53,3

12,0

16,0

48,0

36,0

14,7

48, 0

37,3

6,7

54,7

38,7

33,3

28,0

38,7

100

Entre as famílias da comunidade rural, o nível de SAN esteve associado de forma significativa $(p \leq 0,005)$ com o consumo alimentar de legumes e verduras cozidas, frutas, leite e/ou derivados, cereais e pães, refrigerantes e sucos industrializados (Tabela 4).

Com os alimentos considerados saudáveis (feijão e/ou leguminosas, leite e/ou derivados, cereais e/ou pães, tubérculos e carnes), o consumo não foi diminuído à medida que se elevava a insegurança alimentar. Porém, com exceção dos grupos alimentares dos legumes e verduras cruas/cozidas e das frutas, no caso o consumo foi sendo diminuído nas famílias com insegurança moderada/grave, sendo considerado então um consumo inadequado (entre uma e quatro vezes na semana).

O consumo adequado (entre cinco e sete vezes na semana) de legumes e verduras cozidas e de frutas, e o consumo adequado (entre uma e sete vezes na semana) de leite e/ou derivados e cereais e pães mostrou-se associado com o grau de segurança alimentar e nutricional.

Isso pode estar relacionado ao fato de ser a comunidade Lamarão uma área rural produtora de diversos tipos de alimentos (frutas, hortaliças, leguminosas, trigo), e assim facilitar o acesso físico e a disponibilidade de frutas e hortaliças para as famílias, aliado ao fato de existirem muitas famílias de agricultores, que produzem também para o autoconsumo. Além disso, as famílias de 
trabalhadores da fazenda de orgânicos recebem, mensalmente, doação de uma cesta com hortaliças e frutas da fazenda, o que pode auxiliar o acesso e consumo desses alimentos, sem custo financeiro para as famílias.

No estudo de Carneiro et al. ${ }^{(22)}$, os autores relatam a existência de baixo consumo de hortaliças pelas famílias da comunidade Lamarão, o que foi justificado pelos autores como consequência da cultura alimentar de grande parte da população local, que é formada por pessoas vindas de outras regiões brasileiras. Ao contrário dos dados encontrados no estudo de Carneiro et al. ${ }^{(22)}$, o presente estudo do Lamarão mostrou dados atuais que confirmam consumo adequado de frutas e hortaliças pelas famílias entrevistadas.

No que se refere aos alimentos considerados não saudáveis (embutidos, biscoitos doces/doces, biscoitos salgados/salgadinhos, sucos industrializados), a prevalência de consumo inadequado (entre uma e sete vezes na semana) foi mais elevada em famílias com insegurança moderada/grave (Tabela 4). Assim, pode-se observar consumo elevado de alimentos industrializados (processados ou ultraprocessados) ocorrendo em um ambiente rural.

Tabela 4. Situação de segurança alimentar e nutricional segundo o consumo semanal das famílias, de alimentos considerados saudáveis. Núcleo Rural Agrícola Lamarão, Distrito Federal, 2013.

\begin{tabular}{|c|c|c|c|c|c|}
\hline \multirow[b]{2}{*}{ Variáveis } & \multicolumn{2}{|c|}{ SAN/ISAN Leve } & \multicolumn{2}{|c|}{ ISAN Moderada/ } & \multirow[b]{2}{*}{ p-valor $* *$} \\
\hline & $\mathbf{n}$ & $\%$ & $\mathbf{n}$ & $\%$ & \\
\hline \multicolumn{6}{|l|}{ Legumes e Verduras Cruas } \\
\hline Consumo Adequado*** & 21 & 33,8 & 4 & 30,8 & 0,829 \\
\hline Consumo Inadequado $* * *$ & 41 & 66,2 & 9 & 69,2 & \\
\hline \multicolumn{6}{|l|}{ Legumes e Verduras Cozidos } \\
\hline Consumo Adequado & 24 & 38,7 & 2 & 15,4 & 0,005 \\
\hline Consumo Inadequado & 38 & 61,3 & 11 & 84,6 & \\
\hline \multicolumn{6}{|l|}{ Frutas } \\
\hline Consumo Adequado & 31 & 50 & 2 & 15,4 & 0,002 \\
\hline Consumo Inadequado & 31 & 50 & 11 & 84,6 & \\
\hline \multicolumn{6}{|l|}{ Feijão e/ou Leguminosas } \\
\hline Consumo Adequado & 61 & 98,4 & 13 & 100 & 0,645 \\
\hline Consumo Inadequado & 1 & 1,6 & 0 & 0,0 & \\
\hline \multicolumn{6}{|l|}{ Leite e/ou Derivados } \\
\hline Consumo Adequado & 60 & 96,8 & 9 & 69,2 & 0,001 \\
\hline Consumo Inadequado & 2 & 3,2 & 4 & 30,8 & \\
\hline \multicolumn{6}{|l|}{ Cereais e Pães } \\
\hline Consumo Adequado & 59 & 95,2 & 9 & 69,2 & 0,003 \\
\hline Consumo Inadequado & 3 & 4,8 & 4 & 30,8 & \\
\hline
\end{tabular}


$150 / /$

$\begin{array}{lccccc}\text { Consumo Adequado } & 59 & 95,2 & 11 & 84,6 & 0,166 \\ \text { Consumo Inadequado } & 3 & 4,8 & 2 & 15,4 & \end{array}$

\section{Carnes}

$\begin{array}{lccccc}\text { Consumo Adequado } & 61 & 98,4 & 13 & 100 & 0,645 \\ \text { Consumo Inadequado } & 1 & 1,6 & 0 & 0,0 & \end{array}$

Embutidos

Consumo Adequado

Biscoitos/bolachas doces e/ou doces

$$
\text { Consumo Adequado }
$$

Biscoitos/bolachas salgadas,

salgadinhos de pacote

Consumo Adequado

Consumo Inadequado

11

51

Refrigerantes

Consumo Adequado

Consumo Inadequado

Sucos industrializados

Consumo Adequado

Consumo Inadequado

Total

41

62

$$
16,1
$$

83,9

17,8

82,2

$$
0
$$

0,0

0,100

13

100

15,4

0,947

84,6

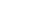

21$$
33,9
$$

66,1

8

61,5

0,005

\begin{tabular}{r}
40,3 \\
59,7 \\
$\mathbf{1 0 0}$ \\
\hline semana \\
$5 x /$ semana
\end{tabular}

**p-valor: $\leq 0,005$;

*** Consumo Adequado de legumes/verduras e frutas: entre 5 a $7 \mathrm{x} /$ semana

Consumo Inadequado de legumes/verduras e frutas: menos que $5 \mathrm{x} /$ semana

Uma provável explicação para essa situação encontra-se na reduzida condição socioeconômica das famílias rurais brasileiras para a compra de alimentos saudáveis e adequados ao consumo, gerando a aquisição de alimentos de menores custos financeiros, porém, de baixa qualidade nutricional, influenciando, dessa forma, outra expressão da insegurança alimentar ${ }^{11,14,15}$. Alia-se a isso a escassez de informações sobre alimentação saudável, ou simplesmente não existir uma educação alimentar e nutricional efetiva que as oriente ${ }^{5,6}$. Nobre et al. ${ }^{(11)}$ encontraram associação estatística entre um menor consumo de hortaliças e suco de frutas em famílias com insegurança alimentar. No estudo de Panigassi et al. ${ }^{25}$, constatou-se consumo elevado de carnes, leite, frutas e hortaliças em famílias com segurança alimentar, sendo o consumo diminuído a medida que se elevava o nível de insegurança alimentar.

Essa tendência de consumo alimentar "urbanizada" é seguida pelos próprios agricultores familiares, os quais passaram a nortear o que e como produzir e o que consumir seguindo essa disposição e exigências derivadas do mercado comercial e fornecedor ${ }^{13}$. 
A própria POF apresentou resultados de consumo da população brasileira pouco saudáveis, como o consumo excessivo e inadequado de embutidos, de variedades de biscoitos salgados e doces, de sucos industrializados e refrigerantes, aliado ao baixo consumo de hortaliças e frutas (menos de $10 \%$ da população consome o recomendado). A combinação do arroz com o feijão, tradicional prato brasileiro, permanece, a despeito do consumo de alimentos inadequados e não saudáveis ${ }^{11}$, como verificado no Lamarão.

Sabe-se que o consumo alimentar inadequado tem consequências no estado nutricional. Em virtude disto, atualmente a ISAN está fortemente associada ao excesso de peso. Pode-se considerar então, que a obesidade e o sobrepeso podem ocorrer como consequência da própria fome $e^{3,5,8,28,32,42}$.

O estudo do Lamarão demonstrou que, nas 53 famílias diagnosticadas com segurança alimentar/ insegurança alimentar leve, residiam 58,5\% de indivíduos adultos com excesso de peso, apontando para o provável comprometimento da qualidade da alimentação da família; entretanto, não foi significativa essa associação estatística entre esses resultados (Tabela 2). O estudo de Santos et al. ${ }^{28}$ já verificou a prevalência de obesidade mais expressiva em adultos de famílias com insegurança alimentar e nutricional, chegando à conclusão que a ISAN estava relacionada não somente à quantidade de alimentos reduzida, mas também à qualidade alimentar inferior, evidenciado pelos excessos de peso. No estudo de Oliveira et al. ${ }^{29}$ com adultos de dois municípios do Nordeste brasileiro, também foi observado que o excesso de peso estava associado com a segurança alimentar/ insegurança alimentar leve das famílias.

Assim, a disponibilidade de alimentos em si não é mais somente a causadora de insegurança alimentar, pois grande parte da população brasileira com ISAN reside em áreas com produção e disponibilidade de alimentos em quantidade suficiente, sem sinais de falta de abastecimento. Logo, o que falta é o acesso a uma alimentação adequada e saudável, sendo ofertados alimentos de qualidade nutritiva, estando relacionado à forma como os alimentos são produzidos, comercializados e consumidos pela população $0^{5,8,13,42}$, pois famílias inseguras podem até ter acesso aos alimentos, no entanto, alimentos com baixa qualidade nutricional.

\section{CONCLUSÃO}

A prevalência da insegurança alimentar e nutricional entre as famílias do Núcleo Rural Agrícola Lamarão demonstra uma possível limitação dessas famílias em relação ao acesso físico e financeiro aos alimentos e à disponibilidade (oferta) de alimentos com qualidade nutritiva.

Os dados socioeconômicos e demográficos (tipo de domicílio e sexo do chefe do domicílio) e os dados de consumo alimentar mostraram-se associados aos níveis de segurança alimentar e nutricional, o que vem comprovar que a garantia da SAN depende dessas questões, que refletem as condições e a qualidade de vida das famílias do Lamarão. 
Conclui-se, com esse estudo, que é importante um olhar aprofundado sob a ótica da garantia da segurança alimentar e nutricional em comunidades rurais. Além disso, é necessário também se considerar as associações entre os fatores socioeconômicos, demográficos e de consumo alimentar, uma vez que são esses fatores que determinam a ocorrência dos diagnósticos de segurança alimentar na população.

Ressalta-se assim que, no Núcleo Rural Lamarão, para a garantia da segurança alimentar e nutricional, é necessária articulação de processos desde o momento da produção de alimentos, passando pelo acesso e oferta a alimentos nutricionalmente adequados às famílias, impactando, consequentemente, no consumo alimentar diário saudável, suficiente e adequado, trazendo mudanças no cenário rural observado. A segurança alimentar e nutricional envolve dimensões e abordagens sociais, econômicas e demográficas familiares que se relacionam estreitamente para a garantia do direito humano à alimentação adequada. Por isso, a SAN deve ser considerada por meio de políticas públicas estruturantes que assegurem esse direito humano a todos.

Esse foi um estudo transversal e muito específico do Distrito Federal, o que impõe certas limitações quanto à extrapolação dos dados observados. Sugere-se, dessa forma, que novos estudos sejam feitos nas outras comunidades rurais do DF e que novos mapeamentos possam assim continuar as análises já realizadas, investigando profundamente as características familiares e os hábitos cotidianos que se relacionem com a garantia da segurança alimentar e nutricional.

\section{REFERÊNCIAS BIBLIOGRÁFICAS}

1- BRASIL. Lei $n^{0}$ 11.346, de 15 de setembro de 2006. Lei de Segurança Alimentar e Nutricional [legislação na internet]. Diário Oficial da União 18 de setembro de 2006 [acesso em 31 mar 2014]. Disponível em http://www4.planalto.gov.br/consea/conferencia/documentos/lei-deseguranca-alimentar-e-nutricional .

2- Instituto Brasileiro de Geografia e Estatística (IBGE). Pesquisa Nacional por Amostra de Domicílios - Segurança Alimentar 2004/2009. Rio de Janeiro: IBGE; 2010.

3- KEPPlE AW, SEGALL-CORRÊA AM. Conceituando e Medindo Segurança Alimentar e Nutricional. Revista Ciência \& Saúde Coletiva 2011; 16(1): 187-199.

4- MALUF RS. Segurança Alimentar e Nutricional e Fome no Brasil - 10 Anos da Cúpula Mundial da Alimentação. Rio de Janeiro: Centro de Referência em Segurança Alimentar e Nutricional - CERESAN; 2006. Relatórios Técnicos 2.

5- KEPPLE AW, GUBERT MB, CORREAA MAS. Instrumentos de Avaliação de Segurança Alimentar e Nutricional. In: Nutrição em Saúde Pública. 1. Ed. Rio de Janeiro: Editora Rubio; 2011. p. 73-97. 
6- BURITY V, FRANCESCHINI T, VALENTE F. Segurança Alimentar e Nutricional e o Direito Humano à Alimentação Adequada. In: Direito Humano à Alimentação Adequada no Contexto da Segurança Alimentar e Nutricional - Módulo 1. Brasília: Ação Brasileira pela Nutrição e Direitos Humanos - ABRANDH, 2010. p. 10-31.

7- $\quad$ LANG RMF, ALMEIDA CCB, TADDEI JAdeA. Segurança Alimentar e Nutricional de Crianças Menores de Dois Anos de Famílias de Trabalhadores Rurais Sem Terra. Ciência \& Saúde Coletiva 2011; 16(7): 3111-3118.

8- MALUF RS, REIS MC. Conceitos e Princípios de Segurança Alimentar e Nutricional. In: Segurança Alimentar e Nutricional - perspectivas, aprendizados e desafios para as políticas públicas. 1. ed. Rio de Janeiro: Editora Fiocruz; 2013. p. 15-42.

9- SOUZA NN, DIAS MdeM, SPERANDIO N, FRANCESCHINI SdoCC, PRIORE SE. Perfil Socioeconômico e Insegurança Alimentar e Nutricional de Famílias Beneficiárias do Programa Bolsa Família no Município de Viçosa, Estado de Minas Gerais, Brasil, em 2011: um Estudo Epidemiológico Transversal. Epidemiologia e Serviços de Saúde 2012; 21(4): 655-662.

10- SEGALL-CORREAA AM, MARIN-LEON L. A Segurança Alimentar no Brasil: Proposição e Usos da Escala Brasileira de Medida da Insegurança Alimentar (EBIA) de 2003 a 2009. Segurança Alimentar e Nutricional, 2009, 16(2), 1-19.

11- NOBRE LN, MURTA, NMG, SOUZA, MM, FERREIRA, NC, CARDOSO, LM, HAMACEK, FR. Segurança Alimentar em uma Comunidade Rural no Alto Vale do Jequitinhonha/ MG. Segurança Alimentar e Nutricional 2009; 16(1): 18-31.

12- Instituto Brasileiro de Geografia e Estatística (IBGE). Pesquisa de Orçamentos Familiares 2008-2009: Análise do Consumo Alimentar Pessoal no Brasil. Rio de Janeiro: IBGE; 2011.

13- MALUF RS, REIS MC. Segurança Alimentar e Nutricional na Perspectiva Sistêmica. In: Segurança Alimentar e Nutricional - perspectivas, aprendizados e desafios para as políticas públicas. 1. ed. Rio de Janeiro: Editora Fiocruz; 2013. p. 43-67

14- ROSA TEC, MONDINI T, GUBERT MB, SATO GS, BENÍCIO MHD`A. Segurança Alimentar em Domicílios Chefiados por Idosos, Brasil. Revista Brasileira de Geriatria e Gerontologia 2012; 15(1): 69-77.

15- VIANNA RPT, SEGALL-CORRÊA AM. Insegurança Alimentar das Famílias Residentes em Municípios do Interior do Estado da Paraíba, Brasil. Revista de Nutrição 2008; 21(Suplemento): 111-122.

16- NASCIMENTO AL, GONÇALVES FCLdaS, MAIA SR, SCHNEIDER S, LIRAS PICde. 
A Construção de Capacidades e Meios de Vida na Garantia da Segurança Alimentar do Rural Pernambucano. Segurança Alimentar e Nutricional 2012; 19(2): 50-62.

17- MENASCHE R, MARQUES FC, ZANETTI C. Autoconsumo e Segurança Alimentar: a Agricultura Familiar a partir dos Saberes e Práticas da Alimentação. Revista de Nutrição 2008; Suplemento 21: 145-158.

18- BOTELHO FBF. As Relações Rural-Urbano no Distrito Federal. Campinas, SP. Tese [Doutorado] - Instituto de Economia; 2001.

19- CALIMAN JF. Caracterização do Potencial Agrícola da Região Rural de Planaltina/DF: Explorando o SISATER. Brasília, DF. Relatório final [Graduação em Gestão do Agronegócio] Universidade de Brasília, Faculdade de Planaltina; 2013.

20- COMPANHIA DO DESENVOLVIMENTO DO PLANALTO CENTRAL (CODEPLAN). Segurança Alimentar e Nutricional no Distrito Federal. Brasília: CODEPLAN; 2012.

21- GHESTI LV. Programa de assentamento dirigido do Distrito Federal - PAD/DF: uma realidade que superou o sonho. Brasília, 2009 [acesso 10 mar 2014]. Disponível em: http://www. coopadf.com.br/o-pad-df.

22- CARNEIRO FF et al. Mapeamento de Vulnerabilidades Socioambientais e de Contextos de Promoção da Saúde Ambiental na Comunidade Rural Do Lamarão, Distrito Federal, 2011. Revista Brasileira de Saúde Ocupacional 2012; 37(125): 143-148.

23- Associação Brasileira de Empresas e Pesquisa (ABEP). Critério de Classificação Econômica Brasil. 2012. Dados com Base no Levantamento Sócio Econômico 2011 - IBOPE. Disponível em: www.abep.org

24- SEGALL-CORREAA AM et al. Projeto: Acompanhamento e avaliação da segurança alimentar de famílias brasileiras: validação de metodologia e de instrumento de coleta de informação. Campinas: Departamento de Medicina Preventiva e Social, Universidade Estadual de Campinas/ Organização Pan-Americana da Saúde/Ministério de Saúde; 2003. Relatório Técnico.

25- PANIGASSI G et al. Insegurança Alimentar Intrafamiliar e Perfil de Consumo de Alimentos. Revista de Nutrição, Campinas. 2008; Suplemento 21: 135-144

26- ANSCHAU FR, MATSUO T, SEGALL-CORRÊA AM. Insegurança Alimentar entre Beneficiários de Programas de Transferência de Renda. Revista de Nutrição 2012; 25(2): 177-189.

27- MARIN-LEON L, FRANCISCO PMSB, SEGALL-CORREAA AM, PANIGASSI G. Bens de Consumo e Insegurança Alimentar: Diferenças de Gênero, Cor de Pele Autorreferida e Condição 
Socioeconômica. Revista Brasileira de Epidemiologia 2011; 14(3): 398-410.

28- SANTOS JV, GIGANTE DP, DOMINGUES MR. Prevalência de Insegurança Alimentar em Pelotas, Rio Grande do Sul, Brasil, e Estado Nutricional de Indivíduos que Vivem nessa Condição. Cadernos de Saúde Pública 2010; 26(1):41-49.

29- OLIVEIRA JS et al. Estado Nutricional e Insegurança Alimentar de Adolescentes e Adultos em duas Localidades de Baixo Índice de Desenvolvimento Humano. Revista de Nutrição 2009; 22(4): 453-465.

30- Ministério da Saúde. Protocolos do Sistema de Vigilância Alimentar e Nutricional - SISVAN na Assistência à Saúde. Ministério da Saúde, Secretaria de Atenção à Saúde, -Departamento de Atenção Básica. Brasília: Ministério da Saúde; 2008.

31- SLATER B, PHILIPPI ST, MARCHIONI DML, FISBERG RM. Validação de Questionários de Frequência Alimentar - QFA: considerações metodológicas. Revista Brasileira de Epidemiologia 2003; 6(3).

32- MOURA EC, SILVA SAda, MALTA DC, NETO OLM. Fatores de Risco e Proteção para Doenças Crônicas: Vigilância por Meio de Inquérito Telefônico, VIGITEL, Brasil, 2007. Cadernos de Saúde Pública 2011; 27(3): 486-496.

33- AIRES JS, MARTINS MC, JOVENTINO ES, XIMENES LB. (In)Segurança Alimentar em Famílias de Pré-Escolares de uma Zona Rural do Ceará. Acta Paulista de Enfermagem 2012; 25(1): 102-108.

34- MONDINI L, ROSA TE, GUBERT MB, SATO GS, BENÍCIO MHD`A. Insegurança Alimentar e Fatores Sociodemográficos Associados nas Áreas Urbana e Rural Do Brasil. Informações Econômicas 2011, 41(2): 52-60.

35- SALLES-COSTA R et al. Associação Entre Fatores Socioeconômicos e Insegurança Alimentar: Estudo de Base Populacional na Região Metropolitana do Rio de Janeiro, Brasil. Revista de Nutrição. 2008; 21(Suplemento): 99-109.

36- SEGALL-CORREAAAM. Insegurança Alimentar Medida a partir da Percepção das Pessoas. Estudos Avançados 2007; 21(60).

37- Instituto Brasileiro de Geografia e Estatística (IBGE). Síntese de Indicadores Sociais - uma Análise das Condições de Vida da População Brasileira. Rio de Janeiro: IBGE; 2013.

38- HOFFMANN R. Determinantes da Insegurança Alimentar no Brasil: Análise dos Dados da PNAD de 2004. Segurança Alimentar e Nutricional 2008; 15(1): 49-61. 
39- BURLANDY L. Transferência Condicionada de Renda e Segurança Alimentar e Nutricional. Ciência \& Saúde Coletiva 2007; 12(6): 1441-1451.

40- BRASIL. Decreto $n^{\circ} 6.135$, de 26 de junho de 2007. Dispõe sobre o Cadastro Único para Programas Sociais do Governo Federal e Dá outras Providências [decreto na internet]. Diário Oficial da União 26 de junho de 2007 [acesso em 31 mar 2014]. Disponível em: http://www.mds. gov.br/acesso-a-informacao/legislacao/bolsafamilia/decretos/2007/Decreto $\% 20 \mathrm{n} \% 206135 \% 20$ de\%2026.06.2007.pdf.

41- GUBERT MB, SANTOS LMP. Determinantes da Insegurança Alimentar no Distrito Federal. Comunicação em Ciências da Saúde 2009; 20(2): 143-150.

42- ROCHA CA. A Contribuição da Economia para a Análise de Políticas Públicas de Segurança Alimentar e Nutricional. In: Segurança Alimentar e Nutricional - perspectivas, aprendizados e desafios para as políticas públicas. 1. ed. Rio de Janeiro: Editora Fiocruz; 2013. p. 69-88.

Artigo apresentado em 06/04/14

Artigo aprovado em 18/05/14

Artigo publicado no sistema em 29/06/14 